\title{
Anti-herpes virus activity of polyether antibiotic CP-44161 in vivo
}

\author{
Yukiko Yamagishi $^{1,7}$, Motoi Ueno ${ }^{1,7}$, Chihoko Ueno ${ }^{1,7}$, Akemi Kato ${ }^{1}$, Ryuichi Kanasaki ${ }^{1}$, Bunji Sato ${ }^{1}$, \\ Koji Takakura ${ }^{2}$, Akihiko Fujie ${ }^{1}$, Hidenori Nakajima ${ }^{3}$, Takashi Fujii ${ }^{4}$, Motohiro Hino ${ }^{5}$ and Eisaku Tsujii ${ }^{6}$
}

In the previous study, we discovered a polyether antibiotic CP-44161, which was reported earlier as an anticoccidal agent, as an anti-varicella zoster virus compound. In this study, we demonstrated that CP-44161 had a very strong and broad anti-herpes virus activities against herpes simplex virus type 1 (HSV-1) and type 2 (HSV-2) in vitro. To determine the antiviral activity of CP-44161 in vivo, we examined its effect on the cutaneous HSV-2 infection model in Balb/c mice. CP-44161 showed inhibitory effect on lesion development as well as acyclovir (ACV) when the treatment was started from day 3 . Meanwhile, in case the start of treatment was delayed until day 4, when ACV was no longer effective, the effectiveness of CP-44161 still remained. In this model, CP-44161 also showed inhibitory effect on the proliferation of HSV-2 DNA in dorsal root ganglia. This is the first article to report that polyether antibiotics can be effective on viral infection in vivo.

The Journal of Antibiotics (2009) 62, 95-98; doi:10.1038/ja.2008.18; published online 23 January 2009

Keywords: antiviral; herpes virus; CP-44161; polyether

\section{INTRODUCTION}

There are eight human herpesvirus that cause various diseases in humans. Herpes simplex virus type 1 (HSV-1), a causative agent of cold sore and corneal herpes, HSV type 2 (HSV-2), a causative agent of genital herpes, and varicella zoster virus (VZV), a causative agent of varicella (chickenpox) and herpes zoster (shingles), belong to alphaherpesvirinae. They usually infect mucosal epithelial cells and travel along the neuron (by a process called retrograde transport) to trigeminal ganglia and then establish latent infection. ${ }^{1}$ Most of the problems with herpesvirus infections are due to reactivation that may lead to recurrent infections. The risk of diseases increases with age, and also frequently occurs in immunocompromised hosts, such as cancer patients, transplantation patients or acquired immunodeficiency syndrome patients. ${ }^{2}$

Standard antiviral drugs at present used in the treatment of HSV and VZV infections include acyclovir (ACV), valaciclovir (VACV, the oral prodrug of $\mathrm{ACV}$ ), famciclovir (the oral prodrug of penciclovir) and vidarabine (Ara-A). ${ }^{3,4}$ Despite a number of recent therapeutic advancements, there remains an urgent need to develop a new class of therapy, especially novel anti-herpes virus agent with a strong potency against HSV-2 and VZV, because anti-herpes virus agents used at present exhibit lower potency against HSV-2 and VZV. Furthermore, as they had some problems, such as cross-resistance and mutagenicity, the development of new agents with different mechanism of action from nucleoside analogs was required.
To discover a new candidate for anti-herpes virus agent, we screened microbial fermentation samples. In the preceding article, ${ }^{5}$ we have reported the discovery of anti-VZV activity of CP-44161 (Figure 1; refs 6,7 ), from an extract of fermentation broth of Dactylosporangium sp. no. 642788. CP-44161, which was a polyether reported earlier as an anticoccidal compound, exhibited a strong anti-VZV activity and lower cytotoxicity than other antiviral polyethers, such as nigericin and monensin.

In this study, we showed strong and broad antiviral activities of CP-44161 against HSV-1 and HSV-2 in vitro. Moreover, we demonstrated the antiviral activity of CP-44161 in vivo on the cutaneous HSV-2 infection model in Balb/c mice.

\section{MATERIALS AND METHODS}

Cells and viruses

African green monkey kidney cell line, Vero (CCL-81), was obtained from the American Type Culture Collection (ATCC; Rockville, MD, USA). Cells were grown and maintained in Eagle's minimal essential medium supplemented with $10 \%$ heat-inactivated fetal bovine serum (FBS), $0.075 \% \mathrm{NaHCO}_{3}, 100 \mathrm{U} \mathrm{ml}^{-1}$ penicillin and $100 \mu \mathrm{g} \mathrm{ml}^{-1}$ streptomycin sulfate. Cells were grown at $37^{\circ} \mathrm{C}$ in an atmosphere of $5 \% \mathrm{CO}_{2}$.

Herpes simplex virus type 1 (VR-3 strain) was kindly provided by Dr T Suzutani (Fukushima Medical University) and HSV-2 (186 strain) ${ }^{8}$ was kindly provided by Dr Y Nishiyama (Nagoya University). Vero cells were

\footnotetext{
${ }^{1}$ Fermentation Research Labs, Astellas Pharma Inc., Tokodai, Tsukuba-shi, Ibaraki, Japan; ${ }^{2}$ Pharmacology Research Labs, Astellas Pharma Inc., Miyukigaoka, Tsukuba-shi, Ibaraki, Japan; ${ }^{3}$ Molecular Medicine Research Labs, Astellas Pharma Inc., Tokodai, Tsukuba-shi, Ibaraki, Japan; ${ }^{4}$ Kikuchi Research Center, The Chemo-Sero-Therapeutic Research Institute, Kyokushi, Kikuchi-shi, Kumamoto, Japan; ${ }^{5}$ Fermentation and Biotechnology Labs, Astellas Pharma Inc., Nakagawara, Kiyosu-shi, Aichi, Japan and ${ }^{6}$ Pharmacology Research Labs, Astellas Pharma Inc., Kashima, Yodogawa-ku, Osaka, Japan

${ }^{7}$ These authors contributed equally to this work.

Correspondence: Dr Y Yamagishi, Fermentation Research Labs., Astellas Pharma Inc., 5-2-3, Tokodai, Tsukuba-shi, Ibaraki, 300-2698, Japan.

E-mail: yukiko.yamagishi@jp.astellas.com
}

Received 2 October 2008; accepted 5 December 2008; published online 23 January 2009 


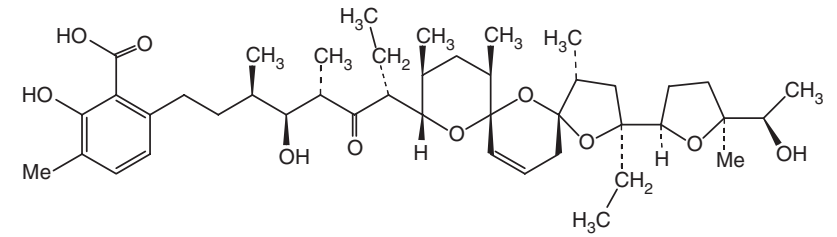

Figure 1 The structure of CP-44161.

used to produce virus pools, quantification of viral titers of stocks by the plaque assay. Viral replication studies of HSV were also performed in Vero cells.

\section{Reagents and antiviral compounds}

CP-44161 was prepared from a culture broth of Dactylosporangium sp. no. 642788 as described in our earlier report. ${ }^{5}$

Adenine 9- $\beta$-D-arabinofuranoside (Ara-A), DMSO and 3-(4,5-dimethylthiazol-2-yl)-2,5,-diphenyltetrazolium bromide (MTT) were obtained from Sigma (St Louis, MO, USA). ACV was purchased from GlaxoSmithKline (Tokyo, Japan). All compounds were dissolved in DMSO and then diluted with cell culture medium to yield $0.1 \%$ DMSO.

\section{In vitro antiviral assays}

The antiviral effects of compounds were determined by plaque reduction assay. Vero cells were grown to confluence in 24-well tissue culture plates and then infected with HSV-1 VR-3 strain or HSV-2 186 strain (40 plaque forming units (PFU) per well). After $1 \mathrm{~h}$ incubation, media containing $1.5 \%$ agarose in the presence or absence of various concentrations of the compounds were added. After 2 days, the cells were stained overnight with $0.01 \%$ neutral red and the number of viral plaques was counted.

\section{Cytotoxicity assay}

The cytotoxicity of compounds against growing Vero cells $\left(5 \times 10^{3}\right.$ cells per well in 96-well tissue culture plates) for $48 \mathrm{~h}$ was determined with an MTT assay as the procedure described earlier. ${ }^{9}$

\section{In vivo studies}

All animal experimental procedures were approved by the Animal Experiment Committee of Fujisawa Pharmaceutical Co. Ltd., the company that is now known as Astellas Pharma Inc.

\section{Antiviral effect on cutaneous HSV infection in Balb/c mice} Seven-week-old female Balb/c mice (Charles River Laboratories, Yokohama, Tokyo, Japan) were used. Each group of animals, controls or treated, contained 10 mice.

Mice were anaesthetized with pentobarbital $\left(50 \mathrm{mg} \mathrm{kg}^{-1}\right.$, i.p.), and then the mid-flank and right foot were clipped and depilated with a chemical depilatory, Hair Remover Milkycream (Kanebo Co. Ltd., Tokyo, Japan). Three days later, HSV-2 $\left(3 \times 10^{3} \mathrm{PFU}\right.$ in $\left.10 \mu \mathrm{l}\right)$ was inoculated on the shin of the right hind paw.

For topical treatments of CP-44161,3\% olive oil solution was formulated. The olive oil without CP-44161 was used as the control. Drug treatment of animals began 3 or 4 days post-infection, and drugs were applied twice a day up to 8 days post-infection with a sterile cotton swab. ACV was dissolved in physiological saline and was administered orally at a dose of $100 \mathrm{mg} \mathrm{kg}^{-1}$.

Beginning 1 day after infection, the inoculated site was evaluated and scored daily for 8 days: 0 , no visible change in the inoculation or surrounding tissue; $1-$ 2 , one or two small vesicles on the inoculation site; $3-4$, many vesicles on the inoculation or surrounding tissue; 5-6, mild zosteriform; 7-8, severe zosteriform.

\section{Quantification of HSV DNA in the dorsal root ganglia}

Seven-week-old female Balb/c mice (Charles River Laboratories, Yokohama, Japan) were infected as described above. Drug treatment of animals began 3 days after infection, and drugs were applied twice a day for the subsequent 3 days. Each group of animals, controls or treated, contained nine mice.

Five days post-infection, the dorsal root ganglia (DRG) were dissected from decapitated mice, immediately frozen by dry ice and stored at $-80^{\circ} \mathrm{C}$ until
Table 1 Antiviral activities against HSV-1,2 and cellular cytotoxicity of CP-44161

\begin{tabular}{lccc}
\hline Compound & $\begin{array}{c}\text { HSV-1 VR-3 } \\
E C_{50}\left(\mu g \mathrm{~m}^{-1}\right)^{\mathrm{a}}\end{array}$ & $\begin{array}{c}\text { HSV-2 186 } \\
E C_{50}\left(\mu g \mathrm{~m}^{-1}\right)^{\mathrm{a}}\end{array}$ & $\begin{array}{c}\text { Vero } \\
C_{50}\left(\mu g \mathrm{~m}^{-1}\right)^{\mathrm{b}}\end{array}$ \\
\hline CP-44161 & 0.025 & 0.018 & 0.58 \\
ACV & 0.025 & 1.5 & $>50$ \\
Ara-A & 1.9 & 5.6 & 25 \\
\hline
\end{tabular}

Values provided in this table represent the mean of three independent experiments.

${ }^{a}$ Antiviral activity was determined by plaque assay. $\mathrm{EC}_{50}$ was the concentration that inhibited $50 \%$ of $\mathrm{HSV}$ replication in Vero cells.

${ }^{b}$ Cytotoxic effect was determined by MTT assay. $\mathrm{CC}_{50}$ was the concentration that showed $50 \%$ cytotoxic effects in Vero cells.

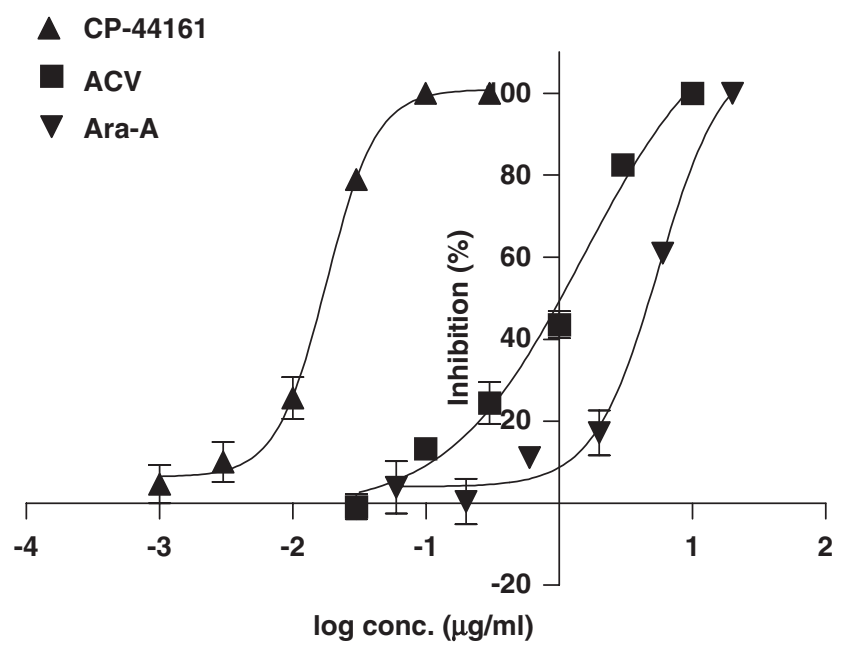

Figure 2 Dose-dependent antiviral activity of CP-44161 against HSV-2. Data are presented as the mean \pm standard deviation of triplicate experiments.

assay. Total DNA in the tissue was extracted with DNeasy tissue kit (Qiagen, Tokyo, Japan) according to a manufacturer's protocol.

The determination of HSV-2 DNA in the total DNA was quantified by realtime PCR using an ABI prism 7900HT sequence detection system (Applied Biosystems, Tokyo, Japan). Primers were designed for the detection of HSV-2 thymidine kinase (TK) gene. The sequences of the primers were as follows: TK (forward), 5'-ggctcctcatatcggggggg-3'; TK (reverse), 5'-cgcggaacagggcaaacagc- 3 '. PCR was performed using the SYBR-green technology. The PCR program consists of activation step at $95^{\circ} \mathrm{C}$ for $10 \mathrm{~min}$ and 40 cycles of denaturation at $94^{\circ} \mathrm{C}$ for $0.5 \mathrm{~min}$, annealing and extension at $67^{\circ} \mathrm{C}$ for $1 \mathrm{~min}$; HSV-2 genome was used as a standard. On each amplification curve, the threshold cycle was determined and used for the quantification of DNA using the ABI prism 7900 software.

\section{Statistical analysis}

Statistical significance was estimated according to $t$-tests using GraphPad Prism 4 software (GraphPad Software Inc.). A $P$-value of $<0.05$ was judged significant.

\section{RESULTS}

Antiviral activities of CP-44161 against HSV-1 and HSV-2

We obtained antiviral activities of CP-44161 against HSV-1 VR-3 strain and HSV-2 186 strain in plaque reduction assay. As shown in Table 1 and Figure 2, CP-44161 inhibited the replication of HSV-1 and HSV-2 in a dose-dependent manner. Of particular interest is the anti-HSV-2 activity (Figure 2). CP-44161 gave an $\mathrm{EC}_{50}$ value of $0.018 \mu \mathrm{g} \mathrm{ml}^{-1}$ compared with an $\mathrm{EC}_{50}$ value of $1.5 \mu \mathrm{g} \mathrm{ml}^{-1}$ for ACV 
and of $5.6 \mu \mathrm{g} \mathrm{ml}^{-1}$ for Ara-A at $48 \mathrm{~h}$. These results showed that CP-44161 had stronger anti-HSV-2 activity than those of ACV and Ara-A, which are in clinical use.

\section{Antiviral effect of CP-44161 on cutaneous HSV-2 infections in Balb/c mice}

Next, we tried to evaluate the antiviral activity of CP-44161 in vivo on the cutaneous HSV-2 infection model in Balb/c mice.

In the preliminary experiment, we found that mice cutaneously infected with HSV-2 showed lesion, but the lesion score began to decrease after day 8 in the control group. Therefore, the therapeutic effect was judged at day 8 . As the oral bioavailability of CP-44161 was poor (data not shown), we evaluated antiviral activity of CP-44161 in vivo using the topical ointment. CP-44161 was administered topically with $3 \%$ olive oil solution (equivalent to $30 \mathrm{mg} \mathrm{kg}^{-1}$ ). ACV was administered orally at a dose of $100 \mathrm{mg} \mathrm{kg}^{-1}$.

In this model, the lesion started to develop on the skin on day 4 (Figures $3 \mathrm{a}$ and $\mathrm{b}$ ). When the treatment was started from day 3 , both CP-44161 and ACV significantly suppressed lesion development in the skin of mice with equivalent efficacy on day 8 (Figure $3 c, P<0.01$ ), but the olive oil control was ineffective in preventing lesion formation. Unlike the case of the treatment started from day 3, when the treatment was started from day 4, ACV was no longer able to inhibit the development of skin lesions. However, CP-44161 showed a significant effect in equivalent efficacy of the results that was started from day 3 (Figure $3 c, P<0.01$ ). These results indicated that $C P-44161$ could be given after the development of symptoms when ACV was no longer effective.

\section{Effect of CP-44161 on HSV-2 DNA in the DRG}

In the above cutaneous infection model, we ascertained the antiviral effect of CP-44161 in vivo by lesion score. To elucidate whether CP44161 could inhibit viral proliferation in vivo, we quantified the HSV2 DNA in DRG. We modified the method for the detection of HSV TK gene by using real-time PCR instead of the PCR analysis. ${ }^{10}$

In the preliminary experiment, we found that HSV proliferation in DRG peaked at day 5 . Therefore, the therapeutic effect was judged at day 5. As shown in Figure 4, 3\% ointment of CP-44161 significantly inhibited the replication of HSV-2 DNA in DRG (Figure $4, P<0.01$ ). It was administered from day 3 to day 5. On day 5, DRG were dissected from treated mice, and the amount of HSV-2 DNA was quantified. When we made a comparison of the effect between CP44161 and ACV on the same condition, CP-44161 exhibited an equivalent efficacy to the oral treatment of ACV $\left(100 \mathrm{mg} \mathrm{kg}^{-1}\right)$ (data not shown).

\section{DISCUSSION}

We demonstrated earlier that the polyether compound CP-44161, discovered from one of the microbial fermentation samples produced by Dactylosporangium sp. no. 642788 , showed anti-VZV activities. ${ }^{5}$

In this study, we first demonstrated that CP-44161 exhibited a broad anti-herpes virus activity against alphaherpesvirinae, such as HSV-1 and HSV-2, in vitro in a dose-dependent manner (Table 1 and Figure 2). CP-44161 inhibited replications of HSV-1 and HSV-2, similar to other polyethers, such as nigericin and monensin (data not shown). Especially in terms of anti-HSV-2 activity, this compound exhibited stronger potency than anti-HSV agents used at present, ACV and Ara-A (Figure 2).

Next we demonstrated that CP-44161 exhibited anti-herpes virus activity in vivo. Owing to its poor oral bioavailability, we evaluated antiviral activity of CP-44161 topically. As the solvent for CP-44161,
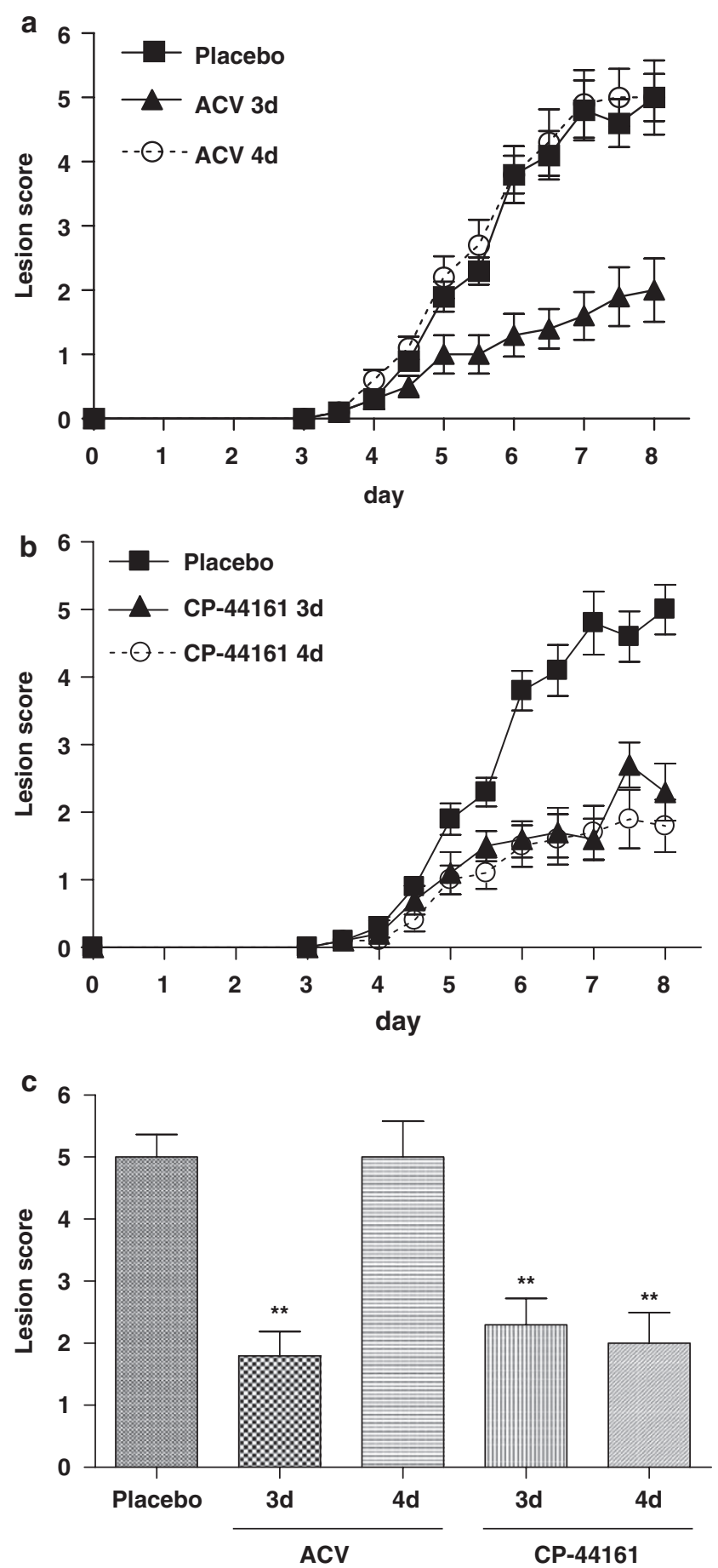

Figure 3 Effects of treatment with CP-44161 and ACV on the development of skin lesions on cutaneous HSV infection in Balb/c mice. Mice were inoculated with $3 \times 10^{3} \mathrm{PFU}$ of HSV-2 strain 186 on the right hind paw on day 0 . (a) ACV $\left(100 \mathrm{mg} \mathrm{kg}^{-1}\right)$ was administered orally twice a day from 3 or 4 days after HSV-2 inoculation. Placebo (saline) was administered orally twice a day from 3 days after HSV-2 inoculation. (b) CP-44161 (3\% ointment) was administered topically twice a day from 3 or 4 days after HSV-2 inoculation. Placebo (olive oil) was administered topically twice a day from 3 days after HSV-2 inoculation. (c) Lesion score on day 8. Data points represent the average score of 10 mice per group \pm s.e. 


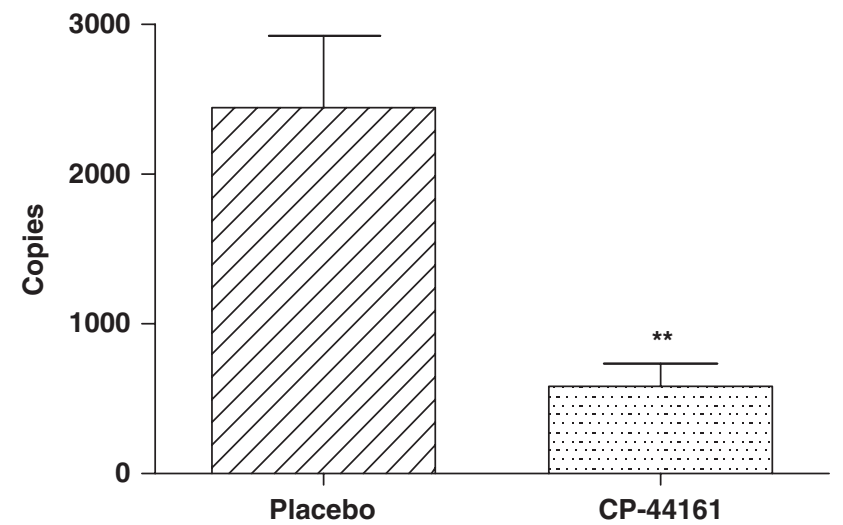

Figure 4 Effects of treatment with CP-44161 on the levels of HSV-2 gene in the dorsal root ganglia (DRG) on cutaneous HSV infection in Balb/c mice. Mice were inoculated with $3 \times 10^{3} \mathrm{PFU}$ of HSV-2 strain 186 on the right hind paw on day 0. CP-44161 (3\% ointment) and placebo (olive oil) was administered topically twice a day from 3 days after HSV-2 inoculation. On day 5, DRG were dissected and DNA was extracted. The copy number of HSV-2 in DRG was quantified by real-time PCR. Data points represent the average score of 9 mice per group \pm s.e.

olive oil was selected, as CP-44161 was dissolved in olive oil completely and the olive oil formulation gave us the best antiviral effect (data not shown). Topical treatment of cutaneous HSV infection has the advantages of convenience and target site selection along with the prospect for maintenance of high local dose rates. However, in herpes zoster, topical monotherapy with ACV is less effective at preventing virus shedding than oral therapy and has little effect on symptoms. ${ }^{11}$ So we selected oral administration of ACV.

The development of a zosteriform rash in this Balb/c mouse model is analogous to the final step in the development of a recrudescent lesion, which might occur after reactivation of latent virus. ${ }^{12}$ In this system, virus traveled from the inoculation site, where virus replicated primarily, to the sensory nervous system and then returned to the skin, resulting in the zosteriform rash. When we started the treatment from day 3, after the onset of lesion development on the skin, both CP-44161 (3\% ointment) and ACV (100 $\left.\mathrm{mg} \mathrm{kg}^{-1}\right)$ showed significant effects in lesion score, with equivalent efficacy on day 8 (Figure 3c). However, CP-44161 was effective even if the start of the treatment delayed, when ACV was no longer effective. Early treatment with antiviral agents, such as ACV and vidarabine, needs to shorten the skin lesions and complications related to herpes zoster. $^{13}$ In clinical practice, the start of administration of VACV limited within the first $72 \mathrm{~h}$ after the onset of symptoms. These results indicated that CP-44161 could be given over $72 \mathrm{~h}$ after the onset; that is, the recalcitrant cases against ACV.

Then we demonstrated that CP-44161 could inhibit viral proliferation in vivo by the quantified detection of the HSV-2 DNA in DRG. The results in Figure 4 confirmed that CP-44161 showed significant inhibition of the replication of HSV-2 DNA in DRG. The result indicated that the inhibition on the proliferation of HSV-2 in DRG may be responsible for the inhibition of zosteriform development of this compound.

Tissue culture studies have demonstrated antiviral activity of polyethers against a wide range of viruses. ${ }^{14-17}$ In terms of the in vivo antiviral activities of polyethers, monensin, narasin, septamycin and nigericin were effective in treating an infection of transmissible gastroenteritis virus in piglets at doses of $0.1-100 \mathrm{mg} \mathrm{kg}^{-1}$ (ref. 18). In Bulgaria, a preparation containing a nigericin derivative (named Pandavir) was used topically as an anti-herpes drug. ${ }^{19}$ However, the mechanism of antiviral activities in vivo has not been characterized. Here, we showed antiviral activity of polyether on murine model for the first time. This enabled us to analyze the mechanism of action of polyethers in vivo easily. Further experimental studies concerning the mode of action of CP-44161 in vitro and in vivo are under investigation.

According to this study, our data indicated that CP-44161 might be a good candidate for a new anti-herpes virus agent. It could be used on not only HSV but also VZV and also might have a potential against post-herpetic neuralgia.

\section{ACKNOWLEDGEMENTS}

We thank Mr R Enomoto for technical advice on Balb/c mice model and Dr K Sudo and Dr K Maki for helpful discussions.

1 Whitley, R. J. \& Roizman, B. Herpes simplex virus infections. Lancet. 357, 1513-1518 (2001).

2 Wutzler, P. Antiviral therapy of herpes simplex and varicella-zoster virus infections. Intervirology 40, 343-356 (1997).

3 Littler, E. The past, present and future of antiviral drug discovery. Idrugs 7, 1104-1112 (2004).

4 De Clercq, E. Recent highlights in the development of new antiviral drugs. Curr. Opin. Microbiol. 8, 552-560 (2005)

5 Yamagishi, Y. et al. Discovery of anti-varicella zoster virus activity of polyether antibiotic CP-44161. J. Antibiot. 62, 89-93 (2009).

6 Celmer, W. D. et al. Polycyclic Ether Antibiotic Produced by New Species of Dactylosporangium in United States Patent 4081532 (Pfizer Inc.: New York, NY, USA, 1976).

7 Tone, J. et al. 18th ICACC Meeting, Atlanta, Georgia Abstract: (1978).

8 Nishiyama, Y. et al. Construction of a US3 lacZ insertion mutant of herpes simplex virus type 2 and characterization of its phenotype in vitro and in vivo. Virology 190, 256-268 (1992).

9 Denizot, F., Lang, R., Rapid colorimetric assay for cell growth and survival. Modifications to the tetrazolium dye procedure giving improved sensitivity and reliability. J. Immunol. Methods 89, 271-277 (1986).

10 Takasaki, I. et al. Allodynia and hyperalgesia induced by herpes simplex virus type-1 infection in mice. Pain 86, 95-101 (2000).

11 Bridges, C. G. et al. The ribonucleotide reductase inhibitor (E)-2'-fluoromethylene2'-deoxycytidine (MDL 101,731): a potential topical therapy for herpes simplex virus infection. Antiviral Res. 27, 325-334 (1995).

12 Simmons, A. \& Nash, A. A. Zosteriform spread of herpes simplex virus as a model of recrudescence and its use to investigate the role of immune cells in prevention of recurrent disease. J. Virol. 52, 816-821 (1984).

13 Whitley, R. J. et al. Disseminated herpes zoster in the immunocompromised host: a comparative trial of acyclovir and vidarabine. The NIAID Collaborative Antiviral Study Group. J. Infect. Dis. 165, 450-455 (1992).

14 Grabley, S. et al. Secondary metabolites by chemical screening. 17. Nigericinol derivatives: synthesis, biological activities, and modeling studies. J. Med. Chem. 35, 939-944 (1992).

15 Johnson, D. C. \& Spear, P. G. Monensin inhibits the processing of herpes simplex virus glycoproteins, their transport to the cell surface, and the egress of virions from infected cells. J. Virol. 43, 1102-1112 (1982).

16 Kousoulas, K. G. et al. Effect of the ionophore monensin on herpes simplex virus type 1 -induced cell fusion, glycoprotein synthesis, and virion infectivity. Intervirology 20, 56-60 (1983).

17 Kaiser, C. J. \& Radsak, K. Inhibition by monensin of human cytomegalovirus DNA replication. Archiv. Virology 94, 229-245 (1987).

18 Gale, C. \& Mcdougald, L. R. Anti-Viral Method in Animals in United States Patent 3995027 (Eli Lilly and Company: Indianapolis, IN, USA, 1976).

19 Dundarov, S. et al. Means for virus disease cure in Japanese Patent 1093524 A (Bulgaria, 1989). 